\section{IN BRIEF}

\author{
REPRODUCTION
}

\section{A sperm cytoskeletal protein that signals oocyte meiotic maturation and ovulation.}

Miller, M. A. et al. Science 291, 2144-2147 (2001)

In many animals, oocytes arrest during meiotic prophase. They are triggered to resume meiosis by sperm, which also cause the gonadal sheath cells (needed for ovulation) to contract. Miller and colleagues show here that the major sperm cytoskeletal protein (MSP) is a signal for both of these events. They go on to show that MSP has a function in sperm locomotion, analogous to that of actin, and conclude that MSP has acquired both extracellular signalling and intracellular cytoskeletal functions during evolution.

\section{PROTEIN METHYLATION}

\section{Arginine methylation of STAT1 modulates IFN $\alpha / \beta$ -} induced transcription.

Mowen, K. A. et al. Cell 104, 731-741 (2001)

Previous analysis of STAT transcription factors has focused on their regulation by phosphorylation, shadowing the importance of another modification - arginine methylation. Here, Mowen and colleagues show that STAT1 is methylated on a conserved arginine residue, a modification mediated by the arginine methyltransferase PRMT1 and required for interferon $\alpha / \beta$ induced transcription. Moreover, they find that the methyltransferase inhibitor MTA inhibits this methylation, leading to increased association of STAT with its inhibitor PIAS1 and so provide a model for the lack of interferon responsiveness observed in certain cancers.

\section{CELL DIVISION}

Requirement of a centrosomal activity for cell cycle progression through $\mathrm{G} 1$ into $\mathrm{S}$ phase

Hinchcliffe, E. H. et al. Science 291, 1547-1550 (2001)

\section{Centrosome-dependent exit of cytokinesis in animal cells}

Piel, M. et al. Science 291, 1550-1553 (2001)

The centrosome - the main microtubule organizing centre in animal cells — has long been thought to change its microtubule organizing properties in response to cell-cycle progression. These two papers now show that the centrosome, in turn, is required at two distinct stages of the cell cycle - the G1 to S transition and the completion of cytokinesis. Piel and colleagues show that, in animal cells, the centrosome is repositioned after anaphase, and is required for microtubule release from the midbody and the completion of cell division. Hinchcliffe et al. removed the centrosome from primate somatic cells, and found that they arrested before $S$ phase, indicating that a centrosomal-associated factor mediates entry into $\mathrm{S}$ phase. They also find that, once in mitosis, the centrosome is not required for the $\mathrm{G} 2$ to $\mathrm{M}$ transition. Together these papers indicate cells may have checkpoints that monitor centrosome duplication or, say the authors, "core centrosomal structures could bind cell cycle regulatory molecules in a way that activates their function".

\title{
Breaking the cycle
}

A broken leg is a minor inconvenience - it may keep you in bed for a few days, but eventually you'll need to get up and carry on as normal. So too, it seems, for yeast with broken DNA. A double-stranded DNA break (DSB) brings the cell cycle to a halt, allowing time for the break to be repaired. After 8-12 hours, however, the cells may escape this arrest even if the DSB persists - in a process known as adaptation.

How can cells do this? James Haber, Marco Foiani and colleagues have investigated this problem and, reporting in Molecular Cell, they show that adaptation is accompanied by a loss of the Rad 53 checkpoint kinase activity.

The DNA-damage checkpoint in Saccharomyces cerevisiae involves a cascade of protein kinases, including Rad53, Chk1, Mec1 and Cdc5 (which is phosphorylated by Rad53 and is known to be involved in adaptation). One way for cells to adapt would be to turn off this kinase cascade, even in the presence of a DSB, so the authors asked what happens to Rad53 and Chk1 in cells that adapt versus those that remain permanently arrested. They chose Rad53 and Chk1 because both are phosphorylated and activated by the upstream kinase Mecl in response to DNA damage.

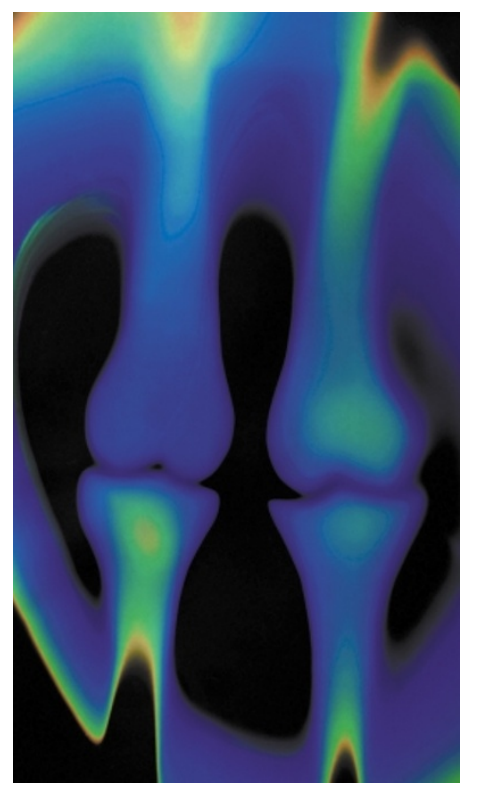

The authors used a site-specific endonuclease to introduce one or two DSBs that could not be repaired. Although cells adapt to one DSB, they remain arrested with two. Interestingly, however, it has been previously shown that yeast cells do not decide to adapt by measuring the number of DSBs per se. Rather, they seem to monitor the amount of single-stranded DNA that is generated as exonucleases nibble back at the broken ends. So deletion of the DNAend-binding protein $y \mathrm{Ku} 70$ prevents adaptation with one DSB, as exonucleases are more free to attack the exposed DNA ends.

Haber, Foiani and colleagues found that, in cells that adapt, there is a concomitant loss of $\operatorname{Rad} 53$ kinase activity. Conversely, in those cells that fail to adapt - because they contain two DSBs or lack functional Cdc5 or yKu70 - the levels of Rad53 activity remain high. When cells adapt, phosphorylation of Chk1 also decreases, indicating that both the Rad53 and Chk1 pathways are involved in adaptation. Moreover, maintenance of the arrested state depends on continued activity of the Mec1 kinase. This means, say the authors, that adaptation is due to inactivation of the kinase cascade itself, rather than, for example, modification of a downstream receptor such that it is no longer sensitive to the cascade.

But why do yeast cells adapt at all? The authors speculate that it may allow the DSB to be repaired in a subsequent cell cycle, and there is evidence that some repair mechanisms may be more efficient during $S$ phase than at other times in the cell cycle. For the moment, though, a next step will be to find out how the kinase cascade is inactivated - by proteolytic destruction of phosphorylated kinases, perhaps, or by dephosphorylation of Rad53 and Chk1?

\section{(2) References and links} ORIGINAL RESEARCH PAPER Pellicioli, A. et al. Regulation of Saccharomyces Rad53 checkpoint kinase during adaptation from DNA damageinduced G2/M arrest. Mol. Cell 7, 293-300 (2001) 


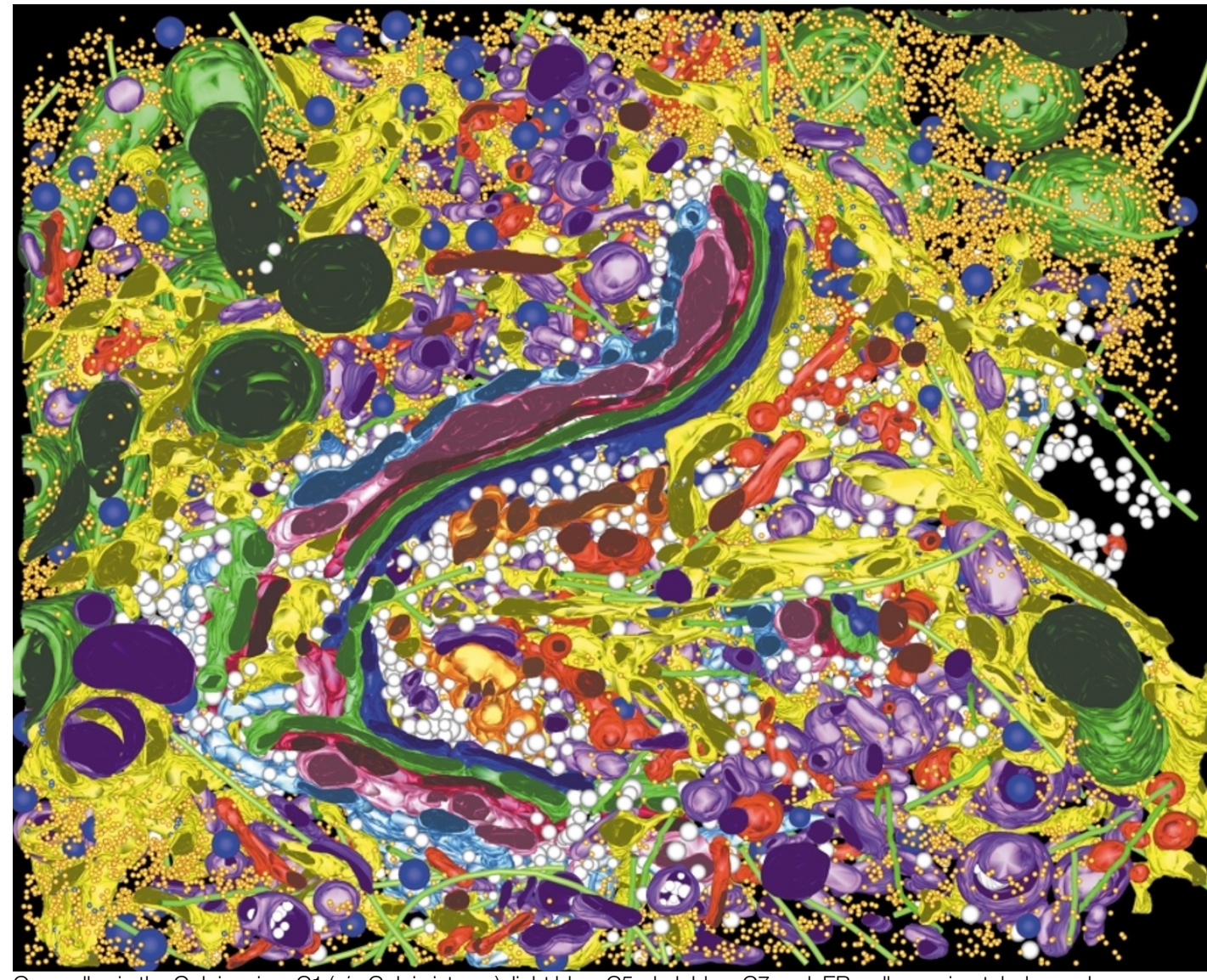

Organelles in the Golgi region. C1 (cis-Golgi cisterna), light blue; C5, dark blue; C7, red; ER, yellow; microtubules and mitochondria, green; dense core vesicles, blue; clathrin-negative vesicles, white; clathrin-positive vesicles and compartments, red; clathrin-negative compartments, purple; free ribosomes, orange. Reproduced with permission from National Academy of Sciences ( 0 (2001).

\section{TECHNIQUE}

\section{The visible cell project ...}

Pancreatic $\beta$-cells (HIT-T15) were put to death through high-pressure freezing and freezesubstitution, cut into ribbons of serial 400-nm-thick sections and studied by electron tomography. Marsh and colleagues collected 480 images of three serial sections by tilting the grid that holds the specimen by $1.5^{\circ}$ over a range of $120^{\circ}$ about two orthogonal axes. They then combined all these images to produce a single, high resolution three-dimensional reconstruction of a $3.1 \times 3.2 \times 1.2 \mu \mathrm{m}^{3}$ area around the Golgi apparatus of a cell. You would think this is a lot of work for a single image, but just look at the result!

The freezing procedure immobilized all cellular activity within milliseconds, so the final image probably reflects the situation found in a live cell. In addition to being incredibly pretty, this snapshot of the cell also gives us some insights into the organization of organelles around the Golgi.

There were not many surprises about the structure of the Golgi itself, which is here made up of seven cisternae ( $\mathrm{C} 1$ to $\mathrm{C} 7$, going from cis to trans Golgi). One interesting observation is that the endoplasmic reticulum (ER) seems to traverse the Golgi stack through aligned openings in the cisternae. There are close contacts between the ER and the C5, C6 and C7 cisternae, which might point to direct exchange between these organelles instead of vesicular transport. Following up on these observations might shed some light on the relationship between the ER and the Golgi, which has been controversial over the past years.

Microtubules seem to follow closely the membranes of the $\mathrm{C} 1$ cisterna of the Golgi and of endosomal compartments. However, the ER seems to be anchored along microtubules at a few points only. Here again, this observation could provide a lead for further investigation into the interaction of organelles with microtubules.

The technique is not only qualitative but also provides a means to quantify organelles in situ in three dimensions, and to measure accurately their physical associations with other organelles. For example, at first sight, you might get the impression that the Golgi region is very crowded, but Marsh and colleagues calculated that only about $34 \%$ of the volume is taken up by organelles, the rest being made up of cytoplasmic matrix.

Although many of the findings in this study are not truly novel or revolutionary, being able to see organelles in three dimensions in their natural cellular context should have high impact on how we imagine life in the cell.

Raluca Gagescu

\section{6) References and links}

ORIGINAL RESEARCH PAPER Marsh, B. J. et al. Organellar relationships in the Golgi region of the pancreatic beta cell line, HIT-T15, visualized by high resolution electron tomography. Proc. Natl Acad. Sci. USA 98, 2399-2406 (2001)
H I G H L I G H T S

\section{WEB WATCH}

... and the virtual cell project

The Virtual Cell is described by its creators as "a general computational framework for modelling cell biological processes". Access to this software is available over the internet through a JAVAbased interface, and although there is no charge for academic use of the Virtual Cell, users are required to register.

So what is the Virtual Cell? It has been developed at the National Resource for Cell Analysis and Modeling (NRCAM), a US-based resource centre supported by the National Center for Research Resources. Those behind it claim that the technology links biochemical and electrophysiological data with experimentally derived microscopic images showing the subcellular localizations of the molecules involved. In this way, they say, "physiological results can be simulated within the empirically derived geometries, thus facilitating the direct comparison of model predictions with experiment".

There are two interfaces to the Virtual Cell - one biologically orientated, the other mathematical. The biological interface has been designed to allow users to define cellular geometry, create models and simulations, and to analyse the results of such simulations.

For those unfamiliar with the technology or software there's a 'User's Guide', backed up by a tutorial designed to work in

conjunction with it, and a 'User Discussion' page for troubleshooting. There are also examples of what the site can do - the applications shown include use of the Virtual Cell to study diffusion processes in mitochondrial cristae, and a simulation of a calcium wave in neuroblastoma cells. Movies are provided with some of the examples, although more explanation would enhance their value.

Alison Mitchell 